\title{
Cytokine Storm in COVID-19: An Overview, Mechanism, Treatment Strategies, and Stem Cell Therapy Perspective
}

\author{
Nuriza Ulul Azmi ${ }^{*}$, Meidi Utami Puteri², Donny Lukmanto ${ }^{3}$ \\ ${ }^{1}$ Laboratory of Pharmacology, Faculty of Pharmacy, Universitas Indonesia, Depok, Indonesia \\ ${ }^{2}$ Department of Experimental Pathology, Graduate School of Comprehensive Human Sciences, Faculty of Medicine, University \\ of Tsukuba, Tsukuba, Japan \\ ${ }^{3}$ Department of Advanced Vision Sciences, Faculty of Medicine, University of Tsukuba; Laboratory of Regenerative Medicine \\ and Stem Cell Biology, Graduate School of Comprehensive Human Sciences, Faculty of Medicine, University of Tsukuba, \\ Tsukuba, Japan
}

all authors contributed equally

\begin{abstract}
Coronavirus disease 2019 (COVID-19) is the disease caused by the highly transmittable novel coronavirus (SARS-CoV-2) infections. The disease was found at the end of December 2019, in Wuhan, China, and quickly spread worldwide with a higher mortality rate compared to the previous coronavirus disease. Cytokine plays an important role in the inflammatory response against coronavirus infection. However, in severely ill patients with COVID-19, an excessive amount of cytokine serum level (cytokine storm) has been associated with the aggravation of coronavirus disease leading to acute respiratory distress syndrome (ARDS). Thus, the management of the cytokine storm could be the key to stop COVID-19 progression. This article reviews an overview related to SARS-CoV-2 and COVID-19, mechanism and prospective treatment strategies for cytokine storm in COVID-19, and a viewpoint of stem cells perspective. The purpose is to provide some information that could be useful for future guidelines in the clinical management of COVID-19.
\end{abstract}

ARTICLE HISTORY

Received: June 2020

Revised: July 2020

Accepted :July 2020

Keywords: COVID-19; SARS-CoV-2; cytokine storm; immunotherapy; stem cell

*corresponding author

Email:nuriza.azmi@farmasi.ui.ac.id

\section{INTRODUCTION}

Coronavirus disease 2019 (COVID-19) was initially emerged in Wuhan, China, in December 2019, and rapidly spread worldwide owing to the explosion of cases. The disease is caused by severe acute respiratory syndrome coronavirus 2 (SARS-CoV-2) that highly transmittable (Lai et al., 2020). As of 15 June 2020, a total of 7,823,289 cases have been reported confirmed for SARS-CoV-2 infection globally, with 431,541 deaths related to COVID-19 (World Health Organization, 2020). The severity of the disease is widely ranged. Some people showed asymptomatic and appeared mild condition, flu-like symptoms, such as fever, dry cough, fatigue, or muscle soreness. However, infected patients could develop to critically ill and even resulting in death. The acute respiratory distress syndrome (ARDS), pneumonia, and multiple organ dysfunction are believed to be some of the severe clinical manifestations of COVID-19 progression (Ye et al., 2020; Singhal, 2020). It has been well understood that cytokine plays an important role in the immune response, e.g., as a defensive mechanism against viral infection (Shimabukuro-Vornhagen et al., 2018). However, the aggravation of the SARS-CoV-2 infection led to the release of excess cytokine (cytokine storm), which could develop into pneumonia (Sun et al., 2020). Of interest, the cytokine storm plays a crucial role in causing ARDS and functional derangement of multi-organ because of an excessive immune response (Chousterman et al., 2017), and it has been reported to be present in critically ill patients with COVID-19 (C. Huang et al., 2020). Therefore, controlling or suppressing the cytokine storm in COVID-19 becomes one of the management strategies to prevent COVID-19 progression and save the patient's life. This article reviews the current literature of COVID-19 and SARS-CoV-2, its association with cytokine storm, and current and stem cell-based treatment strategies to inhibit the cytokine storm in patients with COVID-19. This review aimed to provide a useful information for the development of guidance in clinical diagnosis and treatment of COVID-19.

\section{METHODS}

Literature studies were conducted to access Englishlanguage articles that contain information about COVID-19 using Google Scholar, ScienceDirect, and PubMed engine search from the NCBI database during June 2020. COVID-19, SARS-CoV2, stem cells, and cytokines storm were used as the keyword for engine searching. The information about an ongoing clinical trial related to COVID-19 was accessed from ClinicalTrials. gov and NIH.gov. 
The Clinical Manifestation of SARS-CoV-2 Infection SARS-CoV-2 belongs to the Coronaviridae family in the Nidovirales order (Shereen et al., 2020), along with other highly pathogenic coronaviruses, which are severe acute respiratory syndrome coronavirus (SARS-CoV) and Middle East respiratory syndrome coronavirus (MERS-CoV) (Ye et al., 2020). Coronavirus is singlestranded RNA viruses, size ranging from 26 to $32 \mathrm{kbs}$ in length, and Corona represents crown-like spikes on the outer surface of the virus (Shereen et al., 2020).

The first case of highly pathogenic coronavirus is SARS caused by SARS-CoV started in Guangdong, China, in 2002. The SARS spread around the globe and infected more than 8000 individuals and 774 deaths. Later in 2012, another coronavirus, MERS-CoV, reemerged in Saudi Arabia, with a total of 2428 infected individuals and 838 deaths were reported. Genetic characterization revealed that at the nucleic acid level, SARS-CoV-2 has $78 \%$ similarity with SARS-CoV and 50\% with MERS$\mathrm{CoV}$ (Lu et al., 2020; Shereen et al., 2020). Hence, patients infected by SARS-CoV, MERS-CoV, and SARSCoV-2 showed similar severe symptoms that could lead to pneumonia, followed by ARDS and multiple organ failure (Ye et al., 2020). In COVID-19 patients, the major syndrome is fever, cough, lymphocytopenia, and myalgia or fatigue (Guan et al., 2020; C. Huang et al., 2020; R. Huang et al., 2020). Patient with preexisting metabolic condition (R. Huang et al., 2020) and or elderly is reported to be prone to develop breathing difficulty which might lead to ARDS and result in a fatality (C. Huang et al., 2020).

It has been reported that bats are possible to be the critical host transmitting medium of the virus. However, the spreading of the virus from human to human arise as a result of direct contact with infected individuals exposed to coughing, sneezing, respiratory droplets, or aerosols and penetrate via inhalation (Shereen et al., 2020). Recent studies revealed that both SARS-Cov and SARS-CoV-2 utilize the spike glycoprotein (S protein) on its viral envelope to bind to angiotensin-converting enzyme 2 (ACE2) receptor which is expressed on the surface of human cells (Hoffmann et al., 2020; Hofmann \& Pöhlmann, 2004). The virus needs to attach to the host cells membrane to enter the host cells and proceed to the replication, resulting in viral infection to humans. Of note, the ACE2 receptor is widely expressed in various human cells with macrophages. It is thought to be the essential target cells for coronavirus infection (Hamming et al., 2004), hence partially explain the COVID-19 syndrome and the development of ARDS.

The current diagnosis of COVID-19 relies on SARSCoV-2 RNA detection in nasopharyngeal swab specimen by real-time polymerase chain reaction (RT-
PCR) or antibody test, epidemiological history, clinical manifestations, and chest imaging (Guan et al., 2020; C. Huang et al., 2020). Until June 2020, no approved therapy could reliably cure COVID-19.

\section{Ongoing Cinical Trials For COVID-19 Vaccine}

To date, there are no approved vaccines or treatments for COVID-19 (National Institutes of Health, 2020). Hence, multiple biotechnology institutes and pharmaceutical companies in various countries are now trying to develop the vaccines, and some of them have been known registered for the study of the clinical trial. For example, the Institute of Biotechnology, Academy of Military Medical Sciences, PLA of China is developing the Ad5-nCoV vaccine, which is now entering Phase II of clinical trials (NCT04341389), the ModernaTX, USA is developing the mRNA-1273 vaccine, which is now also entering early Phase II of clinical trials (NCT04405076), and the University of Oxford, U.K. is developing the ChAdOx1 nCoV-19 vaccine, which is now entering phase II/III of clinical trials (NCT04400838) (U.S. National Library of Medicine, 2020). However, the world still needs to wait for another couple of months or even years before the vaccines are ready to be widely deployed. While waiting for the vaccines ready to prevent a future outbreak, finding the best treatment in reducing morbidity and mortality of COVID-19 patients are also urgently required. Many clinical trials are now actively conducted worldwide in evaluating the potential therapeutic agents for COVID-19 from currently available agents such as the existed antiviral drugs, the convalescent plasma from recovered COVID19's patients, and the cytokines inhibitors, which is the focus of this review.

\section{Sars-CoV-2 Infection Leads to Cytokine Storms}

Clinical data from severe COVID-19 patients have suggested that the fatal outcome of ARDS caused by SARS-CoV2 infection often results from alveolar injury in the lung and lead to the respiratory and multiple organ failures (Mehta et al., 2020; Sanders et al., 2020). The clinical studies further found that those conditions are associated with the significant increment of cytokine production in the body, which also termed as cytokine release syndrome (CRS) or cytokine storm (Ye et al., 2020). Cytokines, the small proteins, play an essential role in the immune response to inflammation and infection in the body. However, the overproduction of cytokine may cause tissue damages as the compensation of excessive response immune.

The pro-inflammatory responses were reportedly involved in the pathogenesis of coronavirus (SARS-CoV, MERS-CoV, and SARS-COV-2) in severely ill patients as the ARDS to be the leading cause of death (Lau et al., 2013; Law et al., 2005; Mehta et al., 2020). Some studies 
explained that at the early stage of infection, delayed release of cytokines and chemokines occurs. Later, interferons (IFNs) were produced at a low level and following by a rapidly high level of pro-inflammatory cytokines and chemokines to attract the inflammatory cells, resulting in excessive infiltration into lung tissue and causing injury (Cheung et al., 2005; Ye et al., 2020). Therefore, it is suggested that the inflammatory cells may be involved in lung pathology. In the animal study, the rapid replication of SARS-CoV led to the delayed released of IFNs and accompanied by the accumulation of pathogenic inflammatory mononuclear macrophages (IMM). Further, signals were activated, and infected cells produced chemokines to attract more IMM, resulting in an abnormally high level of many pro-inflammatory cytokines and thus aggravating the disease. In the later phase, the IFN-I and other pro-inflammatory cytokines render the $\mathrm{T}$ cells apoptosis, obstructing the viral clearance (Channappanavar et al., 2016).

IFN- $\alpha$ and IFN- $\beta$, the subtypes of IFN-I, are being essential as the first line defender against viral infection through the Janus kinase/signal transducers and activators of transcription (JAK-STAT) pathway (Song et al., 2020). Coronavirus infection could induce a significant but delayed response of IFNs and later trigger high level of cytokines and chemokines, resulting in cytokine storm that rapidly aggravates the disease in severe condition (Ye et al., 2020). The delayed response has been explained by various mechanisms.

The coronavirus encodes protein NSP1, the viral nonstructural protein 1 , that hinders the phosphorylation of STAT1, thus inhibiting the IFNs secretion (Narayanan et al., 2008). STAT1 is known as the transcription factor for interferon-stimulated genes (ISGs) in the antiviral defense program (Wathelet et al., 2007). The structural protein $\mathrm{M}$ (membrane) and $\mathrm{N}$ (nucleocapsid) encoded by a coronavirus, also could inhibit the IFNs signaling by interfering IRF3, the transcription factor for IFN genes (Park \& Iwasaki, 2020). Moreover, a study revealed that SARS-CoV-2 infection released a low IFN-I during the earlier phase while later inducing pro-inflammatory cytokines. COVID-19 patients showed a high level of pro-inflammatory cytokines and chemokine despite undetectable levels of IFN-I (Blanco-Melo et al., 2020). The high level of IFNs was found correlated with disease severity, with mostly detected in severe patients (Park \& Iwasaki, 2020). It is suggested that the IFNs response may be delayed, supported by in vivo study, IFN-I was not detectable until several hours after viral load (Channappanavar et al., 2016). The defect or suppression of IFN induction is related to the age of hosts and caused by the proteolytic degradation of TRAF3, a signaling molecule downstream of pattern recognition receptors (PRRs) required for IFN transcription. The imbalance of pro-inflammatory cytokines and IFN response in aging may be responsible for the pathogenesis of disease (Park \& Iwasaki, 2020).

An in vitro study also demonstrated that the delayed IFNs response could be caused by the SARS-CoV-2 protein, ORF3b, that shown potently antagonized human IFN-I activation (Konno et al, 2020). Supported by a study, the high level of antibodies recognized ORF3b in SARS-CoV-2 infection during the early phase (Hachim et al, 2020). Another theory has been reported that the profile of IFNs release is correlated with STING (stimulator of IFN genes), an adaptor molecule linking to the production of IFNs and NF-kB through the sensing of cytosolic DNA. During early stage of SARS-CoV infection, viral papain protease contained in viral non-structural proteins interact with STING, results in the downstream IFN secretion. In addition, during the second phase of COVID-19, the activation of STING leads to sudden IFN- $\beta$ secretion and finally induces the cytokine storm (Berthelot \& Liote, 2020). Therefore, various mechanisms to evade and suppress IFNs explain the delayed IFNs response in the severe condition of infection. The delayed impact interfere the first line of immune response against viral infection and sudden release of IFNs in the later phase following by overproduction of cytokine and chemokines leading to cytokine storm that involved in aggravation of the disease, ARDS and organ damages.

COVID-19-induced cytokine storm is characterized by elevated serum cytokine conditions, hyper inflammation, cytopenia, the tendency for ARDS, blood clotting, and increased serum concentration of ferritins (Henderson et al., 2020). Cytokine storm is a condition of uncontrollable overproduction of cytokine which thought to be caused by aberrant immune activation, such as activation of macrophages and other antigen-presenting cells in attempt to notify the existence of virus to lymphocytes, synthesis of pro-inflammatory factors during viral RNA amplification inside the host cells, and viral invasion of lymphocytes, evoking lymphocyte apoptosis (W. Luo et al., 2020). One study in China also has been demonstrated that the observation from 3,939 patients that both have mild and severe degree of disease showed changes in circulating leukocyte subsets and cytokine secretion from normal condition (J. Wang et al., 2020). Besides, Huang and colleagues reported that cytokine storms caused by SARS-CoV-2 infection at Wuhan were characterized with increased plasma concentrations of both Th1secreted cytokines (e.g., IL-1 $\beta$, IFN-induced protein-10 (IP-10), macrophage chemoattractant protein-1 (MCP1), and IFN- $\gamma$ ) that promote the inflammatory response, and Th2-secreted cytokines (e.g., IL-4, IL-10) that regulate the anti-inflammatory response. In principle, the production of anti-inflammatory cytokines, mainly 
is IL-10, represents one of the mechanisms involved in balancing pro-inflammatory responses (Tisoncik et al., 2012). However, some studies have revealed that even though IL-10 is recognized as an anti-inflammatory cytokine, it has positive correlation during lung fibrosis when it was exposed to the lung in a high concentration and a long time, as increased IL-10 expression was found to induce collagen production and fibrocyte recruitment into the lung (Sun et al., 2011). Remarkably, the balance of pro- and anti-inflammatory cytokines play an important role for maintaining lung immune homeostasis, thus miss regulation one or more of these mechanisms will lead towards the cytokine storm syndrome.In addition, the high titer of coronavirus and abundance of cytokines could promote the endothelial and epithelial cells apoptosis that further may cause leak of pulmonary vascular and alveolar edema, rendering to hypoxia condition and ARDS (Ye et al., 2020). The cytokine storm is also suggested to cause the extrapulmonary multiple organ-failure ( $\mathrm{H}$. Wang \& Ma, 2008). Hence, the cytokine storm plays an important role in the inflammatory response during coronavirus infections leading to the ARDS and multiple-organ injury and thus causing the severity of disease and death. It suggests the treatment strategies to inhibit cytokine storm during COVID-19 infection to prevent the progression of the disease.

However, the use of inflammatory cytokine levels and leukocytes level for diagnosis and monitoring of cytokine storm in COVID-19 patients in clinical is not yet standardized and currently limited for research purposes only. Therefore, clinicians currently use more common available inflammation biomarkers as an early biomarker, which can predict COVID-19 disease progression such as serum ferritin level, C-reactive protein (CRP) level, and D-dimer level (England et al., 2020; Henderson et al., 2020). Ferritin, an iron storage protein, and C-reactive protein (CRP) are the most widely used biomarker at hospital for monitoring systemic inflammation. They are known as acute phase reactants produced by liver during inflammation states. Some clinical evidence have reported that ferritin and CRP levels in patients with COVID-19 are reportedly higher in patients with severe compared to moderate disease, and in patients who died compared to those that recovered (England et al., 2020; J. Wang et al., 2020)). The other common biomarker being used is the D-dimer level, a fibrin degradation product. It is usually used as a test to check active clot formation and breakdown. Even though not commonly used in the cytokine storm disorders evaluation, D-dimer level has been reported to correlate with disease severity in COVID-19, and the value of D-dimer $>1 \mu \mathrm{g} / \mathrm{mL}$ has remarked as one of the earliest prognostic markers to predict patients with high risk of mortality (England et al., 2020).

\section{Potential of Cytokines Inhibitor for COVID-19 Treatment}

Normally, when the cytokine is released, the function is to give a signal to the immune system to eliminate the virus as well as the infected alveolar cells. However, in some cases, hyperproduction of cytokines will lead to the aggressive immune response and led to the attack to other healthy alveolar cells, which definitely worsens the patient clinical outcome (Mehta et al., 2020; J. Wang et al., 2020; Ye et al., 2020). In addition, vascular permeability is increased by these pro-inflammatory cytokines, which leads to fluid and blood cells hyper-flowing into the alveoli, developing to respiratory failure, and even death in a short time (Ye et al., 2020). Therefore, it is not a surprise that some existed drugs that target the immune response and block the CRS in COVID-19 patients have become a potential target of recent clinical trials.

\section{Ongoing Clinical Trials for Blocking the Cytokine Storm in COVID-19}

The monoclonal antibody or recombinant protein that directed target key inflammatory cytokines are the potential class of drugs to be evaluated for blocking the cytokine storms in COVID-19 therapies. The level of interleukin 2R (IL-2R), interleukin -1 (IL-1), and interleukin 6 (IL-6) were reported to be positively correlated with the disease severity in COVID-19 patients (Chen et al., 2020). In addition, IL-6 was reported to be significantly increased in patients with COVID-19 that showed severe condition compared to non-severe and associated with increased mortality in patients with COVID-19 (Aziz et al., 2020) while IL-1 was reported as biomarkers that associated with severity and fatal outcomes of patients with COVID-19 (J. Wang et al., 2020). Among many cytokines, Interleukin-6 (IL-6) and Interleukin-1 (IL-1) appear to play an essential role in this inflammation dysregulation through interrupting critical inflammation pathways such as JAK-STAT and NF-kB pathways, respectively (Zhong et al., 2020). Therefore, some agents targeting these molecules become a center of attention for the clinical studies.

\section{Interleukin-6 (IL-6) Inhibitors}

IL-6 is a member of the cytokine family that plays a vital role in acute inflammation. It also has functions in cell metabolism, cell differentiation, and immune regulation (McGonagle et al., 2020). Almost all stromal and immune system cells can generate this small polypeptide. The production of IL- 6 can be triggered during host defense mechanisms by infectious or non-infectious inflammation. In the classical cell signaling pathway, IL-6 exerts its functions by binding to the IL-6 receptor (IL-6R) and then activate some intracellular signaling pathways such as JAK-STAT, YAP-TAZ, and PI3K-AKT pathways (Zhang et al., 2020). The activation of these pathways then promotes a more downstream process 
that regulates cell proliferation, cell differentiation, and immune regulation (Liu et al., 2020). At the moment, there are insufficient data from clinical trials using IL-6 inhibitors for COVID-19 patients. However, several IL-6 inhibitors have now undergone the study for clinical trials in patients with COVID-19, such as sarilumab and tocilizumab.

\section{Sarilumab}

Sarilumab is a human IgG1 monoclonal antibody that binds to both soluble and membrane-bound IL-6R; thus could interfere signaling pathway induced by IL-6. It is originally therapy approved by the FDA for rheumatoid arthritis patients (McCarty \& Robinson, 2018). Regeneron Pharmaceutical, in collaboration with Sanofi, now is in phase III of clinical trial testing sarilumab IV injection (under Kezvara brand name) for hospitalized patients with critical COVID-19 (NCT04315298). There are positive trends in the initial results of Phase II indicated by a decreased marker of systemic inflammation such as, C-reactive protein (CRP) (which changed by $-79 \%,-77 \%$, and $-21 \%$ in the sarilumab 400 $\mathrm{mg}$ group, sarilumab $200 \mathrm{mg}$ group, and placebo group, respectively). However, final results are not yet published since more patients are under recruitment (n:2500) for phase III (U.S. National Library of Medicine, 2020).

\section{Tocilizumab}

Tocilizumab is a recombinant humanized monoclonal antibody against IL-6R that is approved by the FDA for rheumatoid arthritis and CRS induced by chimeric antigen receptor T cell (CAR-T) therapy (Alten, 2011; Zhang et al., 2020). Tocilizumab can be delivered by intravenous (IV) or subcutaneous (SQ) injection. Nevertheless, for the CRS therapy, the IV formulation should be used (Liu et al., 2020; P. Luo et al., 2020). One clinical trial study in France $(n=129)$ (NCT04331808) showed that the number of hospitalized COVID-19 patients (moderate to severe) who had died or who needed ventilation in a group receiving tocilizumab IV injection was lower than in the standard of care group. However, the final results of the study have not been published yet (U.S. National Library of Medicine, 2020). Another study of fifteenhospitalized COVID-19 patients in China had reported that under-treatment of tocilizumab for seven days, the serum level of IL-6 tended to increase in the beginning and then decreased after therapy in 10 patients followed with the better clinical outcome. Unfortunately, a persistent increase in IL-6 was observed in four severely critical patients who failed treatment. This study had suggested that tocilizumab seems an effective treatment option in COVID-19 patients with a sign of cytokine storms. However, the study is only limited in a few numbers of patients and only use laboratory parameter as assessment. Thus further study about the effectiveness of tocilizumab in a larger number with more complete clinical assessments is required (P. Luo et al., 2020).

\section{Interleukin-1 (IL-1) Inhibitor}

IL-1 is one of the pro-inflammatory cytokines and the general name for two distinct proteins, IL-1 $\alpha$ and IL-1 $\beta$, which their biological activities are often indistinguishable. Generally, IL-1 has a function in inflammation process during host defense mechanism against infection, become an underlying mechanism of fever and sepsis. The index between effective and toxic functions of IL1 is known to be so narrow, hence the regulation of IL-I in the human body is so tight. A miss regulation of IL-1 will lead to several immune diseases. Similar to IL-6, IL-1 also exerts its function after binding with IL-1 receptor (IL-1R) and known to transduce and activate one of the famous inflammation pathways, the NF-kB pathway (Dinarello, 1997; Zhong et al., 2020).

\section{Anakinra}

The available drugs known could inhibit the binding of IL-1 and IL-1R is anakinra, a recombinant human IL$1 \mathrm{R}$ antagonist. It is originally therapy approved by the FDA for rheumatoid arthritis patients and cryopyrinassociated periodic syndromes (Shakoory et al., 2016). Several clinical trial studies of the anakinra use in COVID-19 patients are ongoing in various countries, yet there are no results have been reported (U.S. National Library of Medicine, 2020).

\section{Corticosteroids}

For the treatment of COVID-19 inducedhyperinflammation, the attention also goes to the corticosteroids (i.e., prednisone, prednisolone, methylprednisolone, dexamethasone) which have potent anti-inflammatory and antineoplastic functions. Corticosteroids have been commonly used for several disease therapy, such as allergy, autoimmune-related diseases, asthma, and chronic obstructive pulmonary disease (Rice et al., 2017). Therefore, it might be useful to treat COVID-19 patients with acute lung injury and ARDS caused by hyper inflammation in the lung (Mehta et al., 2020). The minimum cost and the availability of corticosteroids have added the potential of drugs to use during this pandemic. Several reports have suggested the positive results of corticosteroids used, remarkably in severe hospitalized COVID-19 patients (Sanders et al., 2020; Tang et al., 2020). Some studies for the clinical trials are ongoing; thereby, the final data have not been published yet (U.S. National Library of Medicine, 2020). However, the benefit may also come with the higher side effects, including delayed viral clearance and increased risk of secondary infection which is shown by previous studies in other viral pneumonia cases (Arabi et al., 2018; Ni et al., 2019; Russell et al., 2020). Hence, it is still cautious for using corticosteroids in COVID-19, the treatment must be under tight diagnosis and assessment from a physician and the use of high dose, long duration, and multiple types should be avoided (Tang et al., 2020). 
Promise of Mesenchymal Stem Cells and its Derivative for the Treatment of COVID-19-Induced Cytokine Storms

An alternative therapy in the clinical trial to combat cytokine storm in COVID-19 patients is the use of stem cell-based medicine. Among many stem cells available, Mesenchymal Stromal/Stem Cell (MSC) is thought to be the most suitable one.

MSC is tissue-derived multipotent stem cells with proliferation capability, intrinsic differentiation potential, and produce an abundance of therapeutic growth factor and cytokines (Pittenger et al., 2019). Depending on its local microenvironment, MSC could promote or restrain inflammation through its cytokine secretion onto immune sytem. Under activated immune system, MSC would release pro-inflammatory cytokine to promote inflammation, but when the immune system is overactivated, MSC would switch to secrete antiinflammatory cytokine to suppress the immune effector cells and activate the immune suppressor cells (Gao et al., 2016; Jiang \& Xu, 2020). According to International Society of Cell Therapy, MSC is minimally defined to possess three features: (1) plastic-adherent fibroblastlike morphology, (2) differentiation into "mesenchymal" tissue lineages (mainly bone, fat, and cartilage), and (3) presence or absence of specific cell surface markers (Dominici et al., 2006). Of note, MSC-based cell therapies have shown to be safe and promising for the treatment of ARDS in early clinical trial setting (Zheng et al., 2014). Hence, MSC-based treatment is expected to be capable of not only act as an immunomodulator but also to repair and regenerate lung tissue in COVID-19 patients with respiratory complications (Chrzanowski et al., 2020).

In addition, Leng and colleagues reported the first pilot of MSC transplantation in COVID-19 patients in China with promising results (Leng et al., 2020). Specifically, 7 out of 10 patients received intravenous infusions of clinical-grade allogeneic MSC, while the remaining three patients received saline as a placebo control group. After 14-day observation, seven MSC-treated patients were recovered whereas two of three patients in the placebotreated group (all placebo-treated patient is reported to have a pre-existing condition) developed ARDS or expired. Notably, a drop in systemic inflammation in the MSC-treated group was observed with 10-fold lower CRP level, lower TNF- $\alpha$ serum level, and increased IL10 serum concentration when compared with the placebo group (Leng et al., 2020). While the result of this study suggested that MSC treatment might be safe as suggested with improvement of symptom and oxygen saturation level within 2-4 days after MSC transplantation with no hypersensitivity and no secondary infection were observed and effective for COVID-19-induced cytokine storm, it is essential to note that this study is limited by its small number of included patient and lack of adequate control groups.

In addition, the MSC product also has some limitations. As therapeutic, MSC could contact the recipient blood, so it is imperative that clinical use of MSC is required to pass ISO109993-1/4 about its hemocompatibility/ biocompatibility with blood (Moll et al., 2019). An arising concern on this issue is MSC expression of tissue factor (TF/CD142/coagulation factor III), a glycoprotein that could activate the extrinsic coagulation pathway, which led to instant blood mediated inflammatory reaction (Moll et al., 2019; Moll et al., 2020). Hence, infusions of high TF/CD 142-expressing MSC products would rile up the pro-thrombotic state of COVID-19 patients and other diseases with hypercoagulability characteristics. Another concern is that the lack of standardization and careful characterization of various MSC-products, since MSC isolated from different tissue demonstrated different characteristics, for example: in bone repair capacity (Kimura et al., 2014), TF expression (George et al., 2018) and growth of brain tumor (Akimoto et al., 2013).

Due to the limitation of cell-based therapy, extracellular vesicles (EVs) have recently attracted attention and is emerging as an alternative to cell-based therapy. EV are a heterogeneous group of cell-derived membranous structures comprising exosomes, apoptotic bodies, and microvesicles, which originate from the endosomal system or which are shed from the plasma membrane (van Niel et al., 2018). Notably, recent paradigm shifts now recognize $\mathrm{EV}$ as a means of intercellular communication and involved in multiple biological processes, which made them attractive for diagnostic or therapeutic purposes. Compared to the whole-cell therapy, EVs are thought to have several advantages, such as the lower risk of tumorigenic effects, lower susceptibility to damage by hostile disease microenvironment, contain a combination of genetic cargo (protein, micro RNA, mRNA, etc.), and the possibility for long-term storage. Given the complexion COVID-19 disease, the fact that MSC secretes various cytokine and chemokines in a paracrine manner, and limitation of MSC, severe COVID-19 patients is more amenable to treatment with a pleiotropic agent like MSC-derived EV. Hence, in the recent report, Sengupta et al. reported a prospective non-randomized open-label cohort study on the use of exosome derived from allogeneic bone marrowderived MSC at the early phase of the clinical trial (Sengupta, 2020) in New York. In detail, 24 COVID-19 patients have received a single $15 \mathrm{~mL}$ intravenous dose of clinical-grade exosome derived from MSC. It is noteworthy that $93 \%$ of patients reported having preexisting conditions. The authors reported that there is 


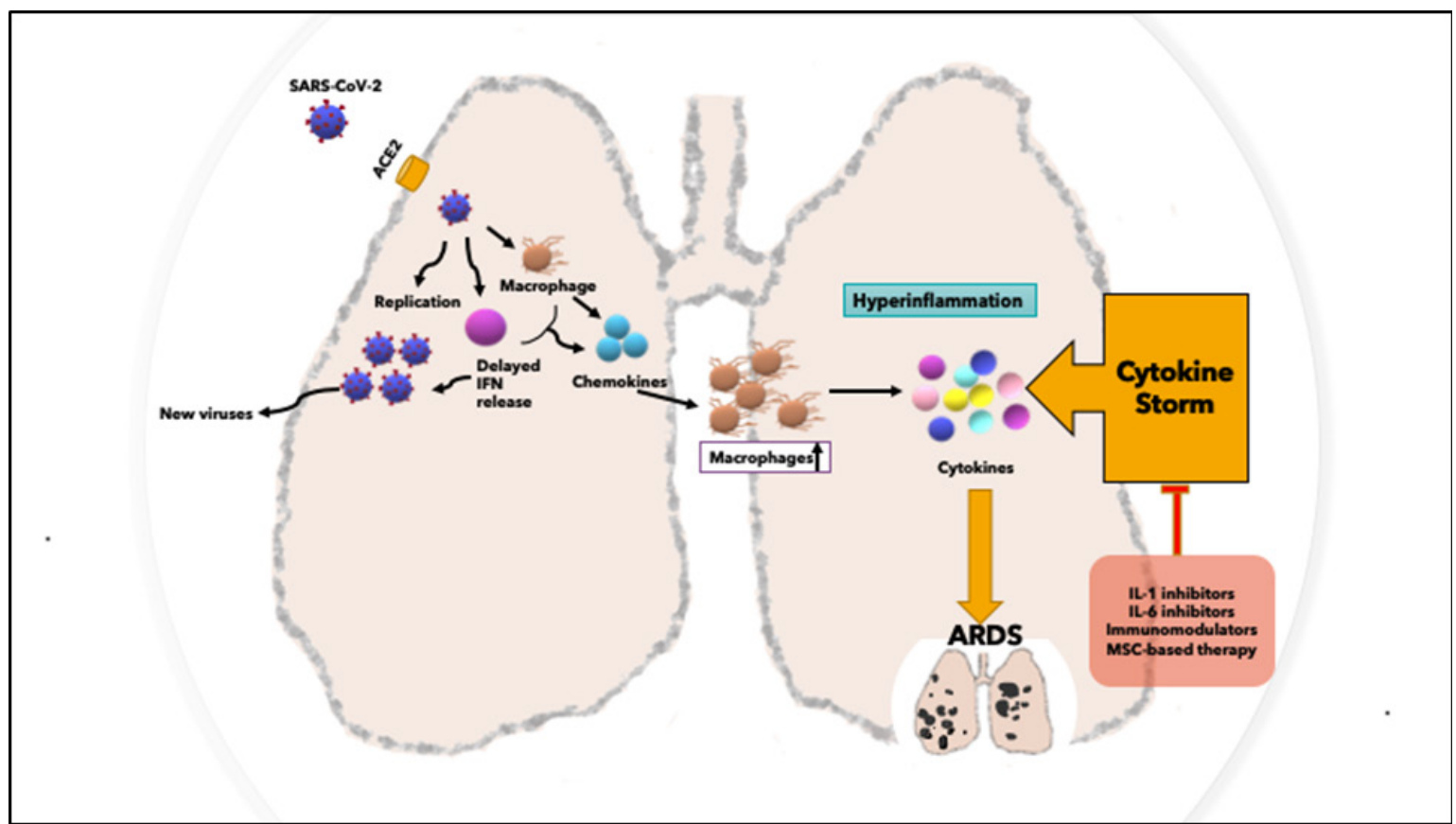

Figure 1. Schematic representation of the mechanism and potential therapy of cytokine storm in COVID-19. SARS-CoV-2 is attached to the ACE2 receptor at the host's cell membrane to enter the host cells, and further perform its replication. Viral infection in the early stage induces delayed IFNs release and accompanied by macrophage accumulation. Later, the chemokine was produced to attract more macrophage, which secretes the high level of cytokines (cytokine storm), leading to ARDS. Suppressing the cytokine storm is the therapy strategies to prevent the severity and mortality progression. SARSCoV-2: severe acute respiratory syndrome coronavirus 2; ACE2: angiotensin-converting enzyme 2; IFN: interferon; ARDS: acute respiratory distress syndrome; IL-1: interleukin 1; IL-6: interleukin 6; MSC: Mesenchymal stem cell.

no adverse effect related to exosome infusion after 72 hours. After 14-day observation, the author reported a survival rate of $83 \%$, with $71 \%$ patients recovered, $13 \%$ patient remained critically ill, and $16 \%$ of patients were expired for reasons unrelated to the treatment. Of importance, the patient's oxygenation was improved, the elevated number of neutrophils counts and improvement of lymphopenia as marked with $192 \%$ increased average pressure of arterial oxygen to fraction of inspired oxygen ratio $(\mathrm{PaO} 2 / \mathrm{FiO} 2)$ and about $42 \%-46 \%$ increase of average $\mathrm{CD} 3+, \mathrm{CD} 4+$, and $\mathrm{CD} 8+$ lymphocyte. On top of that, serum levels of CRP, ferritin, and D-dimer were also reported to be downregulated. Taken together, the result of this study suggested that exosome derived from MSC might be a better promising candidate to treat severe COVID-19 patients owing to its safety, oxygenation restoration properties, and downregulation of cytokine storm marker. However, as with other early report using MSC product, this study is limited by its small sample size, lack of randomization and blinding, only one exosome product were observed, and lack of mechanistic approach of MSC-derived exosome.

Taken together, MSC and exosome derived from MSC might be a promising candidate to treat severe COVID-19 patients owing to its safety and downregulation of cytokine storms marker. Hence, further randomized clinical trials for both MSC-based cell therapy and MSC-derived EV products still needed to validate the early promise of MSC and its derivative for a therapeutic agent for COVID-19 associated cytokine storms.

\section{Concluding Remarks}

COVID-19 is caused by SARS-CoV-2 and quickly became a pandemic threat. We are currently at the beginning of understanding COVID-19 pathophysiology and disease mechanism. Based on the current knowledge, the cytokine storm is closely linked to the COVID-19 in severe conditions. As cytokines play an important role in the inflammatory response, the uncontrollable overproduction of cytokines could aggravate the disease and lead to ARDS and multiple organ failure, even resulting in death. Suppressing the cytokine storm may be the potential to prevent the severity and mortality rate in critically ill patients infected SARS-CoV-2. Thus, a better understanding of cytokine storm-related COVID-19 pathogenesis supports the development of clinical management therapy.

Several clinical trials have been performed to explore the potential therapy of COVID-19 related to cytokine storms, such as inhibitor cytokine production (IL-1 and IL-6 inhibitors), immunomodulator, and stem cell therapy using MSC-based cell therapy and MSC-derived EV 
products (Figure 1). However, further clinical trials still need to be explored to validate the potentials of abovementioned therapies in the management of COVID-19.

\section{REFERENCES}

Akimoto, K., Kimura, K., Nagano, M., Takano, S., Salazar, G. T., Yamashita, T., \& Ohneda, O. (2013). Umbilical cord blood-derived mesenchymal stem cells inhibit, but adipose tissue-derived mesenchymal stem cells promote, glioblastoma multiforme proliferation. Stem Cells and Development, 22(9), 1370-1386. doi:10.1089/scd.2012.0486.

Alten, R. (2011). Tocilizumab: a novel humanized antiinterleukin 6 receptor antibody for the treatment of patients with rheumatoid arthritis. Therapeutic Advances in Musculoskeletal Disease, 3(3), 133-149.

Arabi, Y. M., Mandourah, Y., Al-Hameed, F., Sindi, A. A., Almekhlafi, G. A., Hussein, M. A., . . . Fowler, R. A. (2018). Corticosteroid therapy for critically ill patients with Middle East respiratory syndrome. American Journal of Respiratory and Critical Care Medicine, 197(6), 757-767. doi:10.1164/rccm.201706-1172OC

Aziz, M., Fatima, R., \& Assaly, R. (2020). Elevated interleukin-6 and severe COVID-19: A meta-analysis. Journal of Medical Virology. doi:10.1002/jmv.25948

Berthelot, J. M., \& Liote, F. (2020). COVID-19 as a STING disorder with delayed over-secretion of interferon-beta. EBioMedicine, 56, 102801. doi:10.1016/j.ebiom.2020.102801

Blanco-Melo, D., Nilsson-Payant, B. E., Liu, W. C., Uhl, S., Hoagland, D., Møller, R., . . . tenOever, B. R. (2020). Imbalanced Host Response to SARS-CoV-2 Drives Development of COVID-19. Cell, 181(5), 1036-1045. e1039. doi:10.1016/j.cell.2020.04.026

Channappanavar, R., Fehr, A. R., Vijay, R., Mack, M., Zhao, J., Meyerholz, D. K., \& Perlman, S. (2016). Dysregulated type I interferon and inflammatory monocyte-macrophage responses cause lethal pneumonia in SARS-CoV-infected mice. Cell Host Microbe, 19(2), 181-193. doi:10.1016/j.chom.2016.01.007

Chen, L., Liu, H. G., Liu, W., Liu, J., Liu, K., Shang, J., . . Wei, S. (2020). [Analysis of clinical features of 29 patients with 2019 novel coronavirus pneumonia]. Zhonghua Jie He He Hu Xi Za Zhi, 43(3), 203-208. doi:10.3760/cma.j.issn.1001-0939.2020.03.013

Cheung, C. Y., Poon, L. L., Ng, I. H., Luk, W., Sia, S. F., Wu, M. H., . . . Peiris, J. S. (2005). Cytokine responses in severe acute respiratory syndrome coronavirusinfected macrophages in vitro: possible relevance to pathogenesis. Journal of Virology, 79(12), 7819-7826. doi:10.1128/JVI.79.12.7819-7826.2005

Chousterman, B. G., Swirski, F. K., \& Weber, G. F. (2017). Cytokine storm and sepsis disease pathogenesis. Seminars in Immunopathology, 39(5), 517-528. doi:10.1007/s00281-017-0639-8

Chrzanowski, W., Kim, S. Y., \& McClements, L. (2020). Can stem cells beat COVID-19: Advancing stem cells and extracellular vesicles toward mainstream medicine for lung injuries associated with SARS-CoV-2 infections. Frontiers in Bioengineering and Biotechnology, 8(554). doi:10.3389/fbioe.2020.00554

Dinarello, C. A. (1997). Interleukin-1. Cytokine \& Growth Factor Reviews, 8(4): 253-265.

Dominici, M., Le Blanc, K., Mueller, I., SlaperCortenbach, I., Marini, F. C., Krause, D. S., . . Horwitz, E. M. (2006). Minimal criteria for defining multipotent mesenchymal stromal cells. The International Society for Cellular Therapy position statement. Cytotherapy, 8(4), 315-317. doi:10.1080/14653240600855905

England, J. T., Abdulla, A., Biggs, C. M., Lee, A. Y. Y., Hay, K. A., Hoiland, R. L., . . . Chen, L. Y. C. (2020). Weathering the COVID-19 storm: Lessons from hematologic cytokine syndromes. Blood Reviews, 100707.

Gao, F., Chiu, S. M., Motan, D. A. L., Zhang, Z., Chen, L., Ji, H. L., . . . Lian, Q. (2016). Mesenchymal stem cells and immunomodulation: Current status and future prospects. Cell Death \& Disease, 7(1), e2062-e2062. doi:10.1038/cddis.2015.327

George, M. J., Prabhakara, K., Toledano-Furman, N. E., Wang, Y.-W., Gill, B. S., Wade, C. E., . . . Cox Jr., C. S. (2018). Clinical cellular therapeutics accelerate clot formation. STEM CELLS Translational Medicine, 7(10), 731-739. doi:10.1002/sctm.18-0015

Guan, W.-j., Ni, Z.-y., Hu, Y., Liang, W.-h., Ou, C.-q., He, J.-X., . . . Zhong, N.-s. (2020). Clinical characteristics of coronavirus disease 2019 in China. New England Journal of Medicine, 382(18), 1708-1720. doi:10.1056/ NEJMoa2002032

Hachim, A., Kavian, N., Cohen, C. A., Chin, A. W., Chu, D. K., Mok, C. K. P., . . Valkenburg, S. A. (2020). Beyond the spike: identification of viral targets of the antibody response to SARS-CoV-2 in COVID-19 417 patients. MedRxiv, 20085670. 
Hamming, I., Timens, W., Bulthuis, M., Lely, A., Navis, G., \& van Goor, H. (2004). Tissue distribution of ACE2 protein, the functional receptor for SARS coronavirus. A first step in understanding SARS pathogenesis. The Journal of Pathology, 203(2), 631-637. doi:10.1002/ path. 1570

Henderson, L. A., Canna, S. W., Schulert, G. S., Volpi, S., Lee, P. Y., Kernan, K. F., . . Nigrovic, P. A. (2020). On the alert for cytokine storm: Immunopathology in COVID-19. Arthritis \& Rheumatology, $n / a(\mathrm{n} / \mathrm{a})$. doi:10.1002/art.41285

Hoffmann, M., Kleine-Weber, H., Schroeder, S., Krüger, N., Herrler, T., Erichsen, S., . . . Pöhlmann, S. (2020). SARS-CoV-2 cell entry depends on ACE2 and TMPRSS2 and is blocked by a clinically proven protease inhibitor. Cell, 181(2), 271-280.e278. doi:https://doi. org/10.1016/j.cell.2020.02.052

Hofmann, H., \& Pöhlmann, S. (2004). Cellular entry of the SARS coronavirus. Trends in Microbiology, 12(10), 466-472. doi:10.1016/j.tim.2004.08.008

Huang, C., Wang, Y., Li, X., Ren, L., Zhao, J., Hu, Y., . . . Cao, B. (2020). Clinical features of patients infected with 2019 novel coronavirus in Wuhan, China. The Lancet, 395(10223), 497-506. doi:10.1016/S01406736(20)30183-5

Huang, R., Zhu, L., Xue, L., Liu, L., Yan, X., Wang, J., . . Wu, C. (2020). Clinical findings of patients with coronavirus disease 2019 in Jiangsu province, China: A retrospective, multi-center study. PLOS Neglected Tropical Diseases, 14(5), e0008280. doi:10.1371/ journal.pntd.0008280

Jiang, W., \& Xu, J. (2020). Immune modulation by mesenchymal stem cells. Cell Proliferation, 53(1), e12712. doi:10.1111/cpr.12712

Kimura, K., Nagano, M., Salazar, G. T., Yamashita, T., Tsuboi, I., Mishima, H., . . . Ohneda, O. (2014). The role of CCL5 in the ability of adipose tissue-derived mesenchymal stem cells to support repair of ischemic regions. Stem Cells and Development, 23(5), 488-501. doi:10.1089/scd.2013.0307

Konno, Y., Kimura, I., Uriu, K., Fukushi, M., Irie, T., Koyanagi, Y., . . . Sato, K. SARS-CoV-2 ORF3b is a potent interferon antagonist whoose activity is further increased by a naturally occuring elongation variant. MedRxiv, 088179.

Lai, C. C., Shih, T. P., Ko, W. C., Tang, H. J., \& Hsueh, P. R. (2020). Severe acute respiratory syndrome coronavirus
2 (SARS-CoV-2) and coronavirus disease-2019 (COVID-19): The epidemic and the challenges. International Journal of Antimicrobial Agents, 55(3), 105924. doi:10.1016/j.ijantimicag.2020.105924

Lau, S. K. P., Lau, C. C. Y., Chan, K. H., Li, C. P. Y., Chen, H., Jin, D. Y., . . Yuen, K. Y. (2013). Delayed induction of proinflammatory cytokines and suppression of innate antiviral response by the novel Middle East respiratory syndrome coronavirus: implications for pathogenesis and treatment. Journal of General Virology, 94(Pt 12), 2679-2690. doi:10.1099/vir.0.055533-0

Law, H. K., Cheung, C. Y., Ng, H. Y., Sia, S. F., Chan, Y. O., Luk, W., . . . Lau, Y. L. (2005). Chemokine upregulation in SARS-coronavirus-infected, monocytederived human dendritic cells. Blood, 106(7), 23662374. doi:10.1182/blood-2004-10-4166

Leng, Z., Zhu, R., Hou, W., Feng, Y., Yang, Y., Han, Q., . . . Zhao, R. C. (2020). Transplantation of ACE2mesenchymal stem cells improves the outcome of patients with COVID-19 pneumonia. Aging and disease, 11(2), 216-228. Retrieved from http://www. aginganddisease.org

Liu, B., Li, M., Zhou, Z., Guan, X., \& Xiang, Y. (2020). Can we use interleukin-6 (IL-6) blockade for coronavirus disease 2019 (COVID-19)-induced cytokine release syndrome (CRS)? Journal of Autoimmunity, 111, 102452 .

Lu, R., Zhao, X., Li, J., Niu, P., Yang, B., Wu, H., . . Tan, W. (2020). Genomic characterisation and epidemiology of 2019 novel coronavirus: implications for virus origins and receptor binding. The Lancet, 395(10224), 565-574. doi:10.1016/S0140-6736(20)30251-8

Luo, P., Liu, Y., Qiu, L., Liu, X., Liu, D., \& Li, J. (2020). Tocilizumab treatment in COVID-19: A single center experience. Journal of Medical Virology, 92(7), 814-818.

Luo, W., Li, Y.-X., Jiang, L.-J., Chen, Q., Wang, T., \& Ye, D.-W. (2020). Targeting JAK-STAT signaling to control cytokine release syndrome in COVID-19. Trends in Pharmacological Sciences. doi:10.1016/j. tips.2020.06.007

McCarty, D., \& Robinson, A. (2018). Efficacy and safety of sarilumab in patients with active rheumatoid arthritis. Therapeutic Advances in Musculoskeletal Disease, 10(3), 61-67.

McGonagle, D., Sharif, K., O’Regan, A., \& Bridgewood, C. (2020). The role of cytokines including interleukin-6 in COVID-19 induced pneumonia and macrophage 
activation syndrome-like disease. Autoimmunity Reviews, 19(6), 102537.

Mehta, P., McAuley, D. F., Brown, M., Sanchez, E., Tattersall, R. S., \& Manson, J. J. (2020). COVID-19: Consider cytokine storm syndromes and immunosuppression. The Lancet, 395(10229), 10331034.

Moll, G., Ankrum, J. A., Kamhieh-Milz, J., Bieback, K., Ringdén, O., Volk, H.-D., . . . Reinke, P. (2019). Intravascular mesenchymal stromal/stem cell therapy product diversification: Time for new clinical guidelines. Trends in Molecular Medicine, 25(2), 149-163. doi:https://doi.org/10.1016/j.molmed.2018.12.006

Moll, G., Drzeniek, N., Kamhieh-Milz, J., Geissler, S., Volk, H.-D., \& Reinke, P. (2020). MSC therapies for COVID-19: Importance of patient coagulopathy, thromboprophylaxis, cell product quality and mode of delivery for treatment safety and efficacy. Frontiers in Immunology, 11(1091). doi:10.3389/fimmu.2020.01091

Narayanan, K., Huang, C., Lokugamage, K., Kamitani, W., Ikegami, T., Tseng, C. T., \& Makino, S. (2008). Severe acute respiratory syndrome coronavirus nspl suppresses host gene expression, including that of type I interferon, in infected cells. Journal of Virology, 82(9), 4471-4479. doi:10.1128/JVI.02472-07

National Institutes of Health. (2020). COVID-19 treatment guidelines panel. Coronavirus disease 2019 (COVID-19) treatment guidelines. National Institutes of Health. Retrieved from https://www. covid19treatmentguidelines.nih.gov/ .

Ni, Y. N., Chen, G., Sun, J., Liang, B. M., \& Liang, Z. A. (2019). The effect of corticosteroids on mortality of patients with influenza pneumonia: a systematic review and meta-analysis. Critical Care, 23(1), 99.

Park, A., \& Iwasaki, A. (2020). Type I and Type III Interferons - Induction, Signaling, Evasion, and Application to Combat COVID-19. Cell Host Microbe, 27(6), 870-878. doi:10.1016/j.chom.2020.05.008

Pittenger, M. F., Discher, D. E., Péault, B. M., Phinney, D. G., Hare, J. M., \& Caplan, A. I. (2019). Mesenchymal stem cell perspective: cell biology to clinical progress. NPJ Regenerative Medicine, 4(1), 22. doi:10.1038/ s41536-019-0083-6

Rice, J. B., White, A. G., Scarpati, L. M., Wan, G., \& Nelson, W. W. (2017). Long-term systemic corticosteroid exposure: A systematic literature review. Clinical Therapeutics, 39(11), 2216-2229.
Russell, C. D., Millar, J. E., \& Baillie, J. K. (2020). Clinical evidence does not support corticosteroid treatment for 2019-nCoV lung injury. The Lancet, 395(10223), 473-475.

Sanders, J. M., Monogue, M. L., Jodlowski, T. Z., \& Cutrell, J. B. (2020). Pharmacologic treatments for coronavirus disease 2019 (COVID-19): A review. JAMA, 323(18), 1824-1836.

Sengupta, V. S., Vikram; Lazo, Angel; Woods, Peter; Nolan,Anna and Bremer, Nicholas (2020). Exosomes derived from bone marrow mesenchymal stem cells as treatment for severe COVID-19. Stem Cells and Development, 29(12), 747-754. doi:10.1089/ scd.2020.0080

Shakoory, B., Carcillo, J. A., Chatham, W. W., Amdur, R. L., Zhao, H., Dinarello, C. A., .. . Opal, S. M. (2016). Interleukin-1 receptor blockade is associated with reduced mortality in sepsis patients with features of macrophage activation syndrome: Reanalysis of a prior phase III trial. Critical Care Medicine, 44(2), 275-281.

Shereen, M. A., Khan, S., Kazmi, A., Bashir, N., \& Siddique, R. (2020). COVID-19 infection: Origin, transmission, and characteristics of human coronaviruses. Journal of Advanced Research, 24, 91-98. doi:10.1016/j. jare.2020.03.005

Shimabukuro-Vornhagen, A., Gödel, P., Subklewe, M., Stemmler, H. J., Schlößer, H. A., Schlaak, M., . . . von Bergwelt-Baildon, M. S. (2018). Cytokine release syndrome. Journal for Immunotherapy of Cancer, 6(1), 56. doi:10.1186/s40425-018-0343-9

Singhal, T. (2020). A review of coronavirus disease-2019 (COVID-19). Indian Journal of Pediatrics, 87(4), 281286. doi:10.1007/s12098-020-03263-6

Song, P., Li, W., Xie, J., Hou, Y., \& You, C. (2020). Cytokine storm induced by SARS-CoV-2. Clinica Chimica Acta, 509, 280-287. doi:10.1016/j. cca.2020.06.017

Sun, L., Louie, M. C., Vannella, K. M., Wilke, C. A., LeVine, A. M., Moore, B. B., \& Shanley, T. P. (2011). New concepts of IL-10-induced lung fibrosis: fibrocyte recruitment and $\mathrm{M} 2$ activation in a CCL2/CCR2 axis. The American Journal of Physiology-Lung Cellular and Molecular Physiology, 300(3), L341-353.

Sun, X., Wang, T., Cai, D., Hu, Z., Chen, J., Liao, H., . . . Wang, A. (2020). Cytokine storm intervention in the early stages of COVID-19 pneumonia. Cytokine \& Growth Factor Reviews, 53, 38-42. doi:10.1016/j. cytogfr.2020.04.002 
Tang, C., Wang, Y., Lv, H., Guan, Z., \& Gu, J. (2020). Caution against corticosteroid-based COVID-19 treatment. The Lancet, 395(10239), 1759-1760.

Tisoncik, J. R., Korth, M. J., Simmons, C. P., Farrar, J., Martin, T. R., \& Katze, M. G. (2012). Into the eye of the cytokine storm. Microbiology and Molecular Biology Reviews, 76(1), 16-32.

U.S. National Library of Medicine. (2020). Retrieved from https://clinicaltrials.gov

van Niel, G., D’Angelo, G., \& Raposo, G. (2018). Shedding light on the cell biology of extracellular vesicles. Nature Reviews Molecular Cell Biology, 19(4), 213-228. doi:10.1038/nrm.2017.125

Wang, H., \& Ma, S. (2008). The cytokine storm and factors determining the sequence and severity of organ dysfunction in multiple organ dysfunction syndrome. American Journal of Emergency Medicine, 26(6), 711715. doi:10.1016/j.ajem.2007.10.031

Wang, J., Jiang, M., Chen, X., \& Montaner, L. J. (2020). Cytokine storm and leukocyte changes in mild versus severe SARS-CoV-2 infection: Review of 3939 COVID-19 patients in China and emerging pathogenesis and therapy concepts. Journal of Leukocyte Biology. doi:10.1002/jlb.3covr0520-272r

Wathelet, M. G., Orr, M., Frieman, M. B., \& Baric, R. S. (2007). Severe acute respiratory syndrome coronavirus evades antiviral signaling: role of nsp1 and rational design of an attenuated strain. Journal of Virology, 81(21), 11620-11633. doi:10.1128/JVI.00702-07
World Health Organization. (2020). Coronavirus disease (COVID-19): Situation report - 147. Retrieved from https://www.who.int/emergencies/diseases/novelcoronavirus-2019/situation-reports

Ye, Q., Wang, B., \& Mao, J. (2020). The pathogenesis and treatment of the 'Cytokine Storm' in COVID-19. Journal of Infection, 80(6), 607-613.

Zhang, C., Wu, Z., Li, J. W., Zhao, H., \& Wang, G. Q. (2020). Cytokine release syndrome in severe COVID-19: interleukin-6 receptor antagonist tocilizumab may be the key to reduce mortality. International Journal of Antimicrobial Agents, 55(5), 105954.

Zheng, G., Huang, L., Tong, H., Shu, Q., Hu, Y., Ge, M., ... Xu, J. (2014). Treatment of acute respiratory distress syndrome with allogeneic adipose-derived mesenchymal stem cells: a randomized, placebo-controlled pilot study. Respiratory Research, 15(1), 39. doi:10.1186/14659921-15-39

Zhong, J., Tang, J., Ye, C., \& Dong, L. (2020). The immunology of COVID-19: is immune modulation an option for treatment? The Lancet Rheumatology. doi:10.1016/S2665-9913(20)30120-X 